\title{
Index autorum ad Vol. 6
}

$\mathrm{B}=$ Buchbesprechung - Book R = Berichte

Bayley, N., 125

Bowman, P.H., 247 (B)

Cumming, E., 248 (B)

Fleming, Ch.E., 177

Fröhlich,W.D., 171 (B), 172 (B), 176 (B)

Havighurst, R.J., 247 (B)

Heinrich, K., 105 (B)

Henry, W.E., 248 (B)

Heron, A., 152

Heymann, K., 106 (B)

Hirsch-Ayalon, A., 160 (R)

Homburger, F., 173 (B)

Hountress, P.J., 106 (B)

Kohlberg, L., 11

Korenchevsky, V., 174 (B)

Kraines, R.J., 140

Krüskemper, H., 174 (B), 175 (B)

Kuhlen, R.G., 113

Lang, 173 (B)

Langeveld, M.J., 110 (B)

Lehr, U., 57, 108 (B), 248 (B)

Liddle, G.P., 247 (B)

Loomis, B., 140

Maas, H.S., 34

Review - Livre nouveau V = Varia - Reports - Rapports

Mathiasen, G., 247 (B)

Mathie, O., 176 (B)

Mattews, Ch.U., 247 (B)

Merminod, A., 172 (B)

Mogey,J., 112 (V)

Moore, T., 213

Neugarten, B.L., 140

Noaks, E.H., 247 (B)

Ostrosky, E., 108 (B)

Pierce, J.U., 247 (B)

Puschner, I., 57

Reed, R.B., 175 (B)

Reuchlin, M., 176 (B) 
Ruprecht, 171 (B)

Sontag, L.W., 1

Spitz, R.A, 171 (B)

Stuart, H.C., 175 (B)

Thomae, H, 106 (B), 247 (B), 249 (B)

Ucko, L.E., 213

Valadin, I., 175 (B)

Vandenhoek, 171 (B)

Vliegenhart, W.E., 87

Wood, V., 140 